\title{
Blow-up solutions for the Schrödinger equation in dimension three with a concentrated nonlinearity
}

\section{Solutions explosives de l'équation de Schrödinger en trois dimensions avec une nonlinéarité concentrée}

\author{
Riccardo Adami ${ }^{\mathrm{a}, *}$, Gianfausto Dell'Antonio ${ }^{\mathrm{b}, \mathrm{c}, 1}$, Rodolfo Figari ${ }^{\mathrm{d}}$, Alessandro Teta ${ }^{\mathrm{e}}$ \\ ${ }^{a}$ Département de mathématiques et applications, École normale supérieure, Paris, France \\ ${ }^{\mathrm{b}}$ Dipartimento di Matematica, Università di Roma "La Sapienza", Italy \\ c SISSA-ISAS, Trieste, Italy \\ d Dipartimento di Scienze Fisiche, Università di Napoli "Federico II", Italy \\ e Dipartimento di Matematica Pura e Applicata, Università di L'Aquila, Italy
}

Received 13 August 2002; accepted 10 January 2003

\begin{abstract}
We present some results on the blow-up phenomenon for the Schrödinger equation in dimension three with a nonlinear term supported in a fixed point. We find sufficient conditions for the blow-up exploiting the moment of inertia of the solution and the uncertainty principle. In the critical case, we discuss the additional symmetries of the equation and construct a family of explicit blow-up solutions.

(c) 2004 L'Association Publications de l'Institut Henri Poincaré. Published by Elsevier B.V. All rights reserved
\end{abstract}

\section{Résumé}

On présente des résultats sur le phénomène de l'explosion pour l'équation de Schrödinger en trois dimensions avec un terme nonlinéaire concentré en un point fixé. On trouve des conditions suffisantes pour l'explosion en utilisant le moment d'inertie de la solution et le principe d'indétermination. Dans le cas critique, on met en évidence l'existence de quelques symétries supplémentaires et l'on construit une famille de solutions explosives explicites.

(c) 2004 L'Association Publications de l'Institut Henri Poincaré. Published by Elsevier B.V. All rights reserved

\footnotetext{
* Corresponding author.

E-mail addresses: riccardo.adami@ens.fr (R. Adami), dellantonio@mat.uniroma1.it (G. Dell'Antonio), figari@na.infn.it (R. Figari), teta@univaq.it (A. Teta).

${ }^{1}$ On leave of absence at Centro Linceo Interdisciplinare, Roma, Italy. 


\section{Introduction}

In this paper we study the Schrödinger equation in dimension three with a nonlinearity concentrated in a fixed point.

The analysis of the nonlinearity concentrated in $n$ fixed points was first approached in [2] and [3], in the onedimensional case, while, in the three-dimensional case, results on local and global existence of the solution were given in [1].

Here we shall go further in the analysis considering the problem of the blow-up in the simpler case of a nonlinearity located at the origin. The peculiar feature of this class of nonlinear equations is the fact that they can be reduced to nonlinear Volterra integral equations involving only the time variable and this considerably simplifies the analysis.

As in the linear case ([4]), the equation in dimension three is more singular than the corresponding equation in dimension one. In particular the space of finite energy is strictly larger than the Sobolev space $H^{1}\left(\mathbb{R}^{3}\right)$. Therefore the usual techniques employed for the analysis of the standard NLSE, such as Sobolev inequalities and uncertainty principle (see, e.g., [5-9,11]), cannot be directly applied to the problem treated here.

For the convenience of the reader we recall the position of the evolution problem starting from the linear case.

Let $H_{\alpha}, \alpha \in \mathbb{R}$, be the Schrödinger operator in $L^{2}\left(\mathbb{R}^{3}\right)$ with a point interaction of strength $\alpha$ placed at the origin. It is well known (see, e.g., [4]) that domain and action of $H_{\alpha}$ are given by

$$
\begin{aligned}
& D\left(H_{\alpha}\right)=\left\{u \in L^{2}\left(\mathbb{R}^{3}\right) \mid u=\phi+q G_{0}, \phi \in H_{\mathrm{loc}}^{2}\left(\mathbb{R}^{3}\right), \nabla \phi \in L^{2}\left(\mathbb{R}^{3}\right), \Delta \phi \in L^{2}\left(\mathbb{R}^{3}\right), q \in \mathbb{C},\right. \\
& \left.\lim _{\mathbf{x} \rightarrow 0}\left(u(\mathbf{x})-q G_{0}(\mathbf{x})\right)=\alpha q\right\}, \\
& H_{\alpha} u=-\Delta \phi,
\end{aligned}
$$

where $G_{\lambda}$ denotes the Green function

$$
G_{\lambda}\left(\mathbf{x}-\mathbf{x}^{\prime}\right)=(-\Delta+\lambda)^{-1}\left(\mathbf{x}-\mathbf{x}^{\prime}\right)=\frac{\mathrm{e}^{-\sqrt{\lambda}\left|\mathbf{x}-\mathbf{x}^{\prime}\right|}}{4 \pi\left|\mathbf{x}-\mathbf{x}^{\prime}\right|}, \quad \lambda \geqslant 0 .
$$

For a given smooth real function $\alpha(t), t \in \mathbb{R}$, the solution of the linear nonautonomous evolution problem

$$
\mathrm{i} \frac{\partial \psi}{\partial t}=H_{\alpha(t)} \psi,\left.\quad \psi\right|_{t=0}=\psi_{0}
$$

can be written in the form (see, e.g., [10])

$$
\psi(t, \mathbf{x})=\left[U(t) \psi_{0}\right](\mathbf{x})+\mathrm{i} \int_{0}^{t} \mathrm{~d} s U(t-s ; \mathbf{x}) q(s),
$$

where

$$
q(t)+4 \sqrt{\pi \mathrm{i}} \int_{0}^{t} \mathrm{~d} s \frac{\alpha(s) q(s)}{\sqrt{t-s}}=4 \sqrt{\pi \mathrm{i}} \int_{0}^{t} \mathrm{~d} s \frac{\left[U(s) \psi_{0}\right](0)}{\sqrt{t-s}}
$$

and $U(t)$ is the free unitary group with integral kernel

$$
U\left(t, \mathbf{x}-\mathbf{x}^{\prime}\right)=\frac{\mathrm{e}^{\mathrm{i} \frac{\left|\mathbf{x}-\mathbf{x}^{\prime}\right|^{2}}{4 t}}}{(4 \pi \mathrm{i} t)^{3 / 2}} .
$$

Our nonlinear evolution problem is then defined by taking the strength of the interaction $\alpha(t)$ as a function of the solution itself. Notice that this corresponds to impose a nonlinear boundary condition at the origin (we refer to [1] for details). We fix

$$
\alpha(z)=\gamma z^{\sigma}, \quad z=|q(t)|^{2}, \quad \gamma \in \mathbb{R}, \quad \sigma \in \mathbb{R}^{+} .
$$


With this choice the nonlinear evolution problem is defined by (1.5) where $q(t)$ is the solution of the nonlinear Volterra equation

$$
q(t)+4 \sqrt{\pi \mathrm{i}} \gamma \int_{0}^{t} \mathrm{~d} s \frac{|q(s)|^{2 \sigma} q(s)}{\sqrt{t-s}}=4 \sqrt{\pi \mathrm{i}} \int_{0}^{t} \mathrm{~d} s \frac{\left[U(s) \psi_{0}\right](0)}{\sqrt{t-s}} .
$$

In [1] local existence and uniqueness of the solution of problem (1.5), (1.9) were proven in the space $V$, defined as

$$
\begin{aligned}
V & =\left\{\psi \in L^{2}\left(\mathbb{R}^{3}\right), \psi=\phi+q G_{0}, \phi \in H_{\mathrm{loc}}^{1}\left(\mathbb{R}^{3}\right), q \in \mathbb{C}, \nabla \phi \in L^{2}\left(\mathbb{R}^{3}\right)\right\} \\
& =\left\{\psi \in L^{2}\left(\mathbb{R}^{3}\right), \psi=\phi_{\lambda}+q G_{\lambda}, \phi_{\lambda} \in H^{1}\left(\mathbb{R}^{3}\right), q \in \mathbb{C}, \lambda>0\right\} .
\end{aligned}
$$

Moreover, the $L^{2}$-norm (usually called the mass), and the energy of the solution $\psi(t)=\phi(t)+q(t) G_{0}$

$$
E(\psi(t))=\|\nabla \phi(t)\|_{L^{2}\left(\mathbb{R}^{3}\right)}^{2}+\frac{\gamma}{\sigma+1}|q(t)|^{2 \sigma+2}
$$

are conserved.

Notice that the space $V$ of finite energy is strictly larger than $H^{1}\left(\mathbb{R}^{3}\right)$ and this is the origin of some technical difficulties (see [1]).

From the conservation laws of mass and energy, we managed to prove the following result of global existence.

Theorem 1. For any initial datum $\psi_{0} \in V$, the solution of problem (1.5), (1.9) is global in time if either $\gamma>0$ or $\sigma<1$.

The problem of blow-up arises when the hypotheses of Theorem 1 are not fulfilled. Therefore, from now on we shall always consider $\gamma<0$ and $\sigma \geqslant 1$. Moreover, we shall denote with $I_{\max }=\left(-T_{*}, T^{*}\right)$ the maximal time interval of existence of the solution.

The notion of blow-up for this kind of problems is naturally set looking at Eq. (1.9).

Definition 2. Given the initial datum $\psi_{0} \in V$, the corresponding solution $\psi(t)=\phi(t)+q(t) G_{0}$ is called a blow-up solution if there exists a finite $T_{c}$ such that

$$
\limsup _{t \rightarrow T_{c}}|q(t)|=\infty \text {. }
$$

Using the conservation of the energy, one sees that the above definition is equivalent to the condition

$$
\limsup _{t \rightarrow T_{c}}\|\nabla \phi(t)\|_{L^{2}\left(\mathbb{R}^{3}\right)}=\infty .
$$

It is easily seen that the following blow-up alternative holds: $\psi(t)$ is a blow-up solution if and only if it is not a global solution. Moreover, if $T^{*}$ is finite, then $T_{c}=T^{*}$, whereas if $T_{*}$ is finite, then $T_{c}=-T_{*}$.

In the former case, we will say that the blow-up occurs forward in time, while in the latter we will say that it occurs backward in time.

The rest of the paper is organized as follows.

In Section 2 we introduce the moment of inertia and we compute its first derivative.

In Section 3 we find an expression for the second time derivative of the moment of inertia using an approximation procedure.

In Section 4, exploiting the second derivative of the moment of inertia and a modified version of the uncertainty principle, we derive a sufficient condition on the initial datum to give rise to a blow-up solution. 
In Section 5 we restrict ourselves to the critical case $\sigma=1$ and show the pseudo-conformal invariance of the problem.

In Section 6, exploiting the pseudo-conformal invariance and the family of stationary solutions, we construct a class of explicit blow-up solutions in the critical case.

\section{The first derivative of the moment of inertia}

Following the line of the proof in dimension one ([3]), we shall introduce the moment of inertia, defined as

$$
I(\psi)=\int_{\mathbb{R}^{3}}|\mathbf{x}|^{2}|\psi(\mathbf{x})|^{2} \mathrm{~d} \mathbf{x} .
$$

The first technical result is given in the following lemma.

Lemma 3. If the moment of inertia of the initial datum $\psi_{0}$ is finite, then the associated solution $\psi(t)$ has a finite moment of inertia at any time of existence.

Proof. The moment of inertia of a function $\psi$ can be written as

$$
I(\psi)=\frac{1}{(2 \pi)^{3}} \int_{\mathbb{R}^{3}}\left|\nabla_{\mathbf{k}} \hat{\psi}(\mathbf{k})\right|^{2} \mathrm{~d} \mathbf{k}, \quad \psi \in L^{2}\left(\mathbb{R}^{3}\right),
$$

where

$$
\hat{\psi}(\mathbf{k})=\int_{\mathbb{R}^{3}} \mathrm{~d} \mathbf{x} \mathrm{e}^{i \mathbf{k} \cdot \mathbf{x}} \psi(\mathbf{x})
$$

Denotes the Fourier transform of $\psi$. We make use of the integral representation of the solution $\psi(t)$ proven in [1] (formula (2.20)).

$$
\hat{\psi}(t, \mathbf{k})=\mathrm{e}^{-\mathrm{i}|\mathbf{k}|^{2} t} \hat{\phi}_{0 \lambda}(\mathbf{k})+\frac{q(t)}{|\mathbf{k}|^{2}+\lambda}+\hat{f}_{1 \lambda}(t, \mathbf{k})+\hat{f}_{2 \lambda}(t, \mathbf{k}), \quad \lambda>0,
$$

where $\psi_{0}=\phi_{0 \lambda}+q_{0} G_{\lambda}$

$$
\begin{aligned}
& \hat{f}_{1 \lambda}(t, \mathbf{k})=\frac{\mathrm{i} \lambda}{|\mathbf{k}|^{2}+\lambda} \int_{0}^{t} \mathrm{~d} s \mathrm{e}^{-\mathrm{i}|\mathbf{k}|^{2}(t-s)} q(s), \\
& \hat{f}_{2 \lambda}(t, \mathbf{k})=-\frac{1}{|\mathbf{k}|^{2}+\lambda} \int_{0}^{t} \mathrm{~d} s \mathrm{e}^{-\mathrm{i}|\mathbf{k}|^{2}(t-s)} \dot{q}(s) .
\end{aligned}
$$

Therefore

$$
\begin{aligned}
\nabla_{\mathbf{k}} \hat{\psi}(t, \mathbf{k})= & -2 \mathrm{i} \mathbf{k} t \mathrm{e}^{-\mathrm{i}|\mathbf{k}|^{2} t} \hat{\phi}_{0 \lambda}(\mathbf{k})+\mathrm{e}^{-\mathrm{i}|\mathbf{k}|^{2} t} \nabla_{\mathbf{k}} \hat{\phi}_{0 \lambda}(\mathbf{k})-\frac{2 \mathbf{k}}{\left(|\mathbf{k}|^{2}+\lambda\right)^{2}} q(t) \\
& +\nabla_{\mathbf{k}} \hat{f}_{1 \lambda}(t, \mathbf{k})+\nabla_{\mathbf{k}} \hat{f}_{2 \lambda}(t, \mathbf{k}) .
\end{aligned}
$$

Let us study the r.h.s. of (2.7).

The first term is square integrable since $\phi_{0 \lambda}$ belongs to $H^{1}\left(\mathbb{R}^{3}\right)$; the second term is square integrable as a consequence of the definition of the moment of inertia. The third term is obviously in $L^{2}\left(\mathbb{R}^{3}\right)$. 
Taking the gradient of $\hat{f}_{1 \lambda}(t, \mathbf{k})$ and of $\hat{f}_{2 \lambda}(t, \mathbf{k})$ we have

$$
\begin{aligned}
& \nabla_{\mathbf{k}} \hat{f}_{1 \lambda}(t, \mathbf{k})=-\frac{2 \mathrm{i} \lambda \mathbf{k}}{\left(|\mathbf{k}|^{2}+\lambda\right)^{2}} \int_{0}^{t} \mathrm{~d} s \mathrm{e}^{-\mathrm{i}|\mathbf{k}|^{2}(t-s)} q(s)+\frac{2 \mathbf{k} \lambda}{|\mathbf{k}|^{2}+\lambda} \int_{0}^{t} \mathrm{~d} s \mathrm{e}^{-\mathrm{i}|\mathbf{k}|^{2}(t-s)}(t-s) q(s), \\
& \nabla_{\mathbf{k}} \hat{f}_{2 \lambda}(t, \mathbf{k})=\frac{2 \mathbf{k}}{\left(|\mathbf{k}|^{2}+\lambda\right)^{2}} \int_{0}^{t} \mathrm{~d} s \mathrm{e}^{-\mathrm{i}|\mathbf{k}|^{2}(t-s)} \dot{q}(s)+\frac{2 \mathrm{i} \mathbf{k}}{|\mathbf{k}|^{2}+\lambda} \int_{0}^{t} \mathrm{~d} s \mathrm{e}^{-\mathrm{i}|\mathbf{k}|^{2}(t-s)}(t-s) \dot{q}(s) .
\end{aligned}
$$

Recalling that both $f_{1 \lambda}(t)$ and $f_{2 \lambda}(t)$ belong to $H^{1}\left(\mathbb{R}^{3}\right)$ and $(t-\cdot) q \in H^{3 / 4}(0, T)$ (for the proof see [1], Theorem 6), from (2.8) and (2.9) we conclude that $\nabla_{\mathbf{k}} \hat{f}_{1 \lambda}(t)$ and $\nabla_{\mathbf{k}} \hat{f}_{2 \lambda}(t)$ are both square integrable.

Indeed, the most singular term is the second one in the r.h.s. of (2.9). Following the analysis performed in [1] (Formulas (2.25) and (2.26)), we can conclude that this term belongs to $L^{2}\left(\mathbb{R}^{3}\right)$.

We proceed with the analysis of $I(\psi(t))$, which in the sequel will be simply denoted by $I(t)$, as a function of $t$. Concerning the first derivative of $I(t)$, we have the following result:

Proposition 4. Given $\psi_{0} \in V$ with $I_{0}=\int_{\mathbb{R}^{3}} \mathrm{~d} \mathbf{x}\left|\mathbf{x} \psi_{0}(\mathbf{x})\right|^{2}$, the function $I(t)=\int_{\mathbb{R}^{3}}|\mathbf{x} \psi(t, \mathbf{x})|^{2}$ belongs to $C^{1}\left(\left[0, T^{*}\right)\right)$ and

$$
\dot{I}(t)=\frac{1}{2 \pi^{3}} \operatorname{Im} \int_{\mathbb{R}^{3}} \mathrm{~d} \mathbf{k} \hat{\psi}(t, \mathbf{k}) \mathbf{k} \cdot \nabla_{\mathbf{k}} \overline{\hat{\psi}(t, \mathbf{k})} .
$$

Proof. First we note that the integral in the r.h.s. of (2.10) is finite.

Given $t \in\left[0, T^{*}\right)$, we introduce a regularized version of the moment of inertia:

$$
I_{\varepsilon}(t)=\frac{1}{(2 \pi)^{3}} \int_{\mathbb{R}^{3}} \mathrm{~d} \mathbf{k}\left|\nabla_{\mathbf{k}} \hat{\psi}(t, \mathbf{k})\right|^{2} \mathrm{e}^{-|\mathbf{k}|^{2} \varepsilon} .
$$

We preliminarly observe that, from monotone convergence theorem, one has

$$
I(t)=\lim _{\varepsilon \rightarrow 0} I_{\varepsilon}(t) .
$$

Now we compute the time derivative of $I_{\varepsilon}$.

Applying repeatedly the estimates

$$
\begin{aligned}
& \left|\mathrm{e}^{-\mathrm{i}|\mathbf{k}|^{2}(t+h)}-\mathrm{e}^{-\mathrm{i}|\mathbf{k}|^{2} t}\right| \leqslant|\mathbf{k}|^{2} h+\frac{|\mathbf{k}|^{4} h}{2}, \\
& \left|(t+h) \mathrm{e}^{-\mathrm{i}|\mathbf{k}|^{2}(t+h)}-t \mathrm{e}^{-\mathrm{i}|\mathbf{k}|^{2} t}\right| \leqslant h\left(1+|\mathbf{k}|^{2}\right)+\frac{|\mathbf{k}|^{4} h^{2} t}{2},
\end{aligned}
$$

one shows that (see (2.7), (2.8), (2.9))

$$
\begin{aligned}
& \frac{1}{h}\left|\nabla_{\mathbf{k}} \hat{\psi}(t+h, \mathbf{k})-\nabla_{\mathbf{k}} \hat{\psi}(t, \mathbf{k})\right| \\
& \leqslant|\mathbf{k}|\left[2\left(1+|\mathbf{k}|^{2}\right)+|\mathbf{k}|^{4} h t\right]\left|\hat{\phi}_{0 \lambda}(\mathbf{k})\right|+\left(|\mathbf{k}|^{2}+\frac{1}{2}|\mathbf{k}|^{4} h\right)\left|\nabla_{\mathbf{k}} \hat{\phi}_{0 \lambda}(\mathbf{k})\right|+\frac{2|\mathbf{k}|}{\left(|\mathbf{k}|^{2}+\lambda\right)^{2}}\|q\|_{L^{\infty}(0, t)} \\
& \quad+\frac{2(\lambda+1)|\mathbf{k}|}{|\mathbf{k}|^{2}+\lambda}\left[\frac{2+2|\mathbf{k}|^{2}+|\mathbf{k}|^{4} h}{|\mathbf{k}|^{2}+\lambda}+2+|\mathbf{k}|^{2}+\frac{1}{2}|\mathbf{k}|^{4} h t\right]\|q\|_{W^{1,1}(0, t)} .
\end{aligned}
$$

From (2.15) and the inequality 


$$
\begin{aligned}
& \left.\frac{1}{h}|| \nabla_{\mathbf{k}} \hat{\psi}(t+h, \mathbf{k})\right|^{2}-\left|\nabla_{\mathbf{k}} \hat{\psi}(t, \mathbf{k})\right|^{2} \mid \\
& \quad \leqslant \frac{1}{h}\left|\nabla_{\mathbf{k}} \hat{\psi}(t+h, \mathbf{k})-\nabla_{\mathbf{k}} \hat{\psi}(t, \mathbf{k})\right|\left(\left|\nabla_{\mathbf{k}} \hat{\psi}(t+h, \mathbf{k})\right|+\left|\nabla_{\mathbf{k}} \hat{\psi}(t, \mathbf{k})\right|\right)
\end{aligned}
$$

we find that $\left.\frac{\exp \left(-\left|\mathbf{k}^{2}\right| \varepsilon\right)}{h}|| \nabla_{\mathbf{k}} \hat{\psi}(t+h, \mathbf{k})\right|^{2}-\left|\nabla_{\mathbf{k}} \hat{\psi}(t, \mathbf{k})\right|^{2} \mid$ is estimated by an $L^{1}$ function independent of $h$. Hence one obtains

$$
\dot{I}_{\varepsilon}(t)=\frac{1}{4 \pi^{3}} \operatorname{Re} \int_{\mathbb{R}^{3}} \mathrm{~d} \mathbf{k} \mathrm{e}^{-|\mathbf{k}|^{2} \varepsilon} \nabla_{\mathbf{k}} \hat{\psi}(t, \mathbf{k}) \cdot \partial_{t} \nabla_{\mathbf{k}} \overline{\hat{\psi}(t, \mathbf{k})} .
$$

Since

$$
\partial_{t} \nabla_{\mathbf{k}} \hat{\psi}(t, \mathbf{k})=-2 \mathrm{ik} \hat{\psi}(t, \mathbf{k})-\mathrm{i}|\mathbf{k}|^{2} \nabla_{\mathbf{k}} \hat{\psi}(t, \mathbf{k}),
$$

one has

$$
\dot{I}_{\varepsilon}(t)=\frac{1}{2 \pi^{3}} \operatorname{Im} \int_{\mathbb{R}^{3}} \mathrm{~d} \mathbf{k} \hat{\psi}(t, \mathbf{k}) \mathbf{k} \cdot \nabla_{\mathbf{k}} \overline{\hat{\psi}(t, \mathbf{k})} \mathrm{e}^{-|\mathbf{k}|^{2} \varepsilon} .
$$

To prove (2.10) we now remove the regularizing factor.

From

$$
\hat{\psi}(t, \mathbf{k})=\hat{\phi}_{\lambda}(t, \mathbf{k})+\frac{q(t)}{|\mathbf{k}|^{2}+\lambda}
$$

with $\phi_{\lambda}(t) \in H^{1}\left(\mathbb{R}^{3}\right)$, we have

$$
\hat{\psi}(t, \mathbf{k}) \mathbf{k} \cdot \nabla_{\mathbf{k}} \overline{\hat{\psi}(t, \mathbf{k})}=\hat{g}_{\lambda}(t, \mathbf{k})+q(t) \frac{\mathbf{k}}{|\mathbf{k}|^{2}+\lambda} \cdot \nabla_{\mathbf{k}} \overline{\hat{\phi}_{\lambda}(t, \mathbf{k})},
$$

where we have introduced the notation

$$
\hat{g}_{\lambda}(t, \mathbf{k})=\hat{\phi}_{\lambda}(t, \mathbf{k}) \mathbf{k} \cdot \nabla_{\mathbf{k}} \overline{\hat{\psi}(t, \mathbf{k})}-\frac{2|\mathbf{k}|^{2}}{\left(|\mathbf{k}|^{2}+\lambda\right)^{3}}|q(t)|^{2} .
$$

From the Cauchy-Schwartz inequality, it follows that

$$
\left|\int_{\mathbb{R}^{3}} \mathrm{~d} \mathbf{k} g_{\lambda}(t, \mathbf{k})\right| \leqslant C_{1}\left\|\phi_{\lambda}\right\|_{C^{0}\left([0, t] ; H^{1}\left(\mathbb{R}^{3}\right)\right)}+2\|q\|_{C^{0}([0, t])}^{2} \int_{\mathbb{R}^{3}} \mathrm{~d} \mathbf{k} \frac{|\mathbf{k}|^{2}}{\left(|\mathbf{k}|^{2}+\lambda\right)^{3}} .
$$

Moreover, for any $s \in[0, t]$ we have

$$
\begin{aligned}
\left|\int_{\mathbb{R}^{3}} \mathrm{~d} \mathbf{k} q(t) \frac{\mathbf{k}}{|\mathbf{k}|^{2}+\lambda} \cdot \nabla_{\mathbf{k}} \overline{\hat{\phi}_{\lambda}(s, \mathbf{k})} \mathrm{e}^{-|\mathbf{k}|^{2} \varepsilon}\right| \leqslant & \|q\|_{C^{0}([0, t])}\left|\int_{\mathbb{R}^{3}} \mathrm{~d} \mathbf{k} \nabla_{\mathbf{k}} \cdot\left(\frac{\mathbf{k}}{|\mathbf{k}|^{2}+\lambda} \mathrm{e}^{-|\mathbf{k}|^{2} \varepsilon} \hat{\phi}_{\lambda}(s, \mathbf{k})\right)\right| \\
& +\|q\|_{C^{0}([0, t])} \mid \int_{\mathbb{R}^{3}} \mathrm{~d} \mathbf{k} \hat{\phi}_{\lambda}(s, \mathbf{k}) \nabla_{\mathbf{k}} \cdot\left(\frac{\mathbf{k}}{|\mathbf{k}|^{2}+\lambda} \mathrm{e}^{-|\mathbf{k}|^{2} \varepsilon}\right) .
\end{aligned}
$$

Using divergence theorem one easily shows that the first term is zero. The modulus of the second term in the r.h.s. of (2.24) gives

$$
\left|\int_{\mathbb{R}^{3}} \mathrm{~d} \mathbf{k} \overline{\hat{\phi}_{\lambda}(t, \mathbf{k})} \mathrm{e}^{-|\mathbf{k}|^{2} \varepsilon}\left[\frac{3-2 \varepsilon|\mathbf{k}|^{2}}{|\mathbf{k}|^{2}+\lambda}-\frac{2|\mathbf{k}|^{2}}{\left(|\mathbf{k}|^{2}+\lambda\right)^{2}}\right]\right|
$$




$$
\begin{aligned}
\leqslant & C_{3}\left\|\phi_{\lambda}\right\|_{C^{0}\left([0, t] ; L^{2}\left(\mathbb{R}^{3}\right)\right)}\left(\int_{\mathbb{R}^{3}} \mathrm{~d} \mathbf{k} \frac{1}{\left(|\mathbf{k}|^{2}+\lambda\right)^{2}}\right)^{1 / 2}+C_{4}\left\|\phi_{\lambda}\right\|_{C^{0}\left([0, t] ; L^{2}\left(\mathbb{R}^{3}\right)\right)}\left(\int_{\mathbb{R}^{3}} \mathrm{~d} \mathbf{k} \frac{|\mathbf{k}|^{4}}{\left(|\mathbf{k}|^{2}+\lambda\right)^{4}}\right)^{1 / 2} \\
& +C_{5}\left\|\phi_{\lambda}\right\|_{C^{0}\left([0, t] ; L^{2}\left(\mathbb{R}^{3}\right)\right)}\left(\varepsilon^{2} \int_{\mathbb{R}^{3}} \mathrm{~d} \mathbf{k} \mathrm{e}^{-|\mathbf{k}|^{2} \varepsilon} \frac{|\mathbf{k}|^{4}}{\left(|\mathbf{k}|^{2}+\lambda\right)^{2}}\right)^{1 / 2} .
\end{aligned}
$$

In order to obtain an estimate independent of $\varepsilon$ for the last term in the r.h.s. of (2.25), we observe that

$$
\int_{\mathbb{R}^{3}} \mathrm{~d} \mathbf{k} \mathrm{e}^{-|\mathbf{k}|^{2} \varepsilon} \frac{|\mathbf{k}|^{4}}{\left(|\mathbf{k}|^{2}+\lambda\right)^{2}}<\int_{\mathbb{R}^{3}} \mathrm{~d} \mathbf{k} \mathrm{e}^{-|\mathbf{k}|^{2} \varepsilon}=\left(\frac{\pi}{2 \varepsilon}\right)^{3 / 2}
$$

which shows that the last term in (2.25) can be made arbitrarily small.

Now, we note that

$$
\begin{aligned}
I(t) & =\lim _{\varepsilon \rightarrow 0}\left(I_{\varepsilon}\left(\psi_{0}\right)+\int_{0}^{t} \mathrm{~d} s \dot{I}_{\varepsilon}(s)\right) \\
& =I_{0}+\lim _{\varepsilon \rightarrow 0} \int_{0}^{t} \mathrm{~d} s \int_{\mathbb{R}^{3}} \mathrm{~d} \mathbf{k} \mathrm{e}^{-|\mathbf{k}|^{2} \varepsilon} \hat{\psi}(t, \mathbf{k}) \mathbf{k} \cdot \nabla_{\mathbf{k}} \overline{\hat{\psi}(t, \mathbf{k})} .
\end{aligned}
$$

From (2.23), (2.25) and (2.26) we get an estimate of $\dot{I}_{\varepsilon}$ uniform with respect to $s$ and $\varepsilon$.

Then, by the dominated convergence theorem we can interchange the limit $\varepsilon \rightarrow 0$ with the integration. This concludes the proof of Proposition 4.

\section{Second derivative of the moment of inertia}

For the computation of the second derivative of the moment of inertia we proceed in two steps: we first prove the result for regular data and then we extend it to data in the space of finite energy $V$.

Proposition 5. Given the initial datum $\psi_{0}=\phi_{0 \lambda}+q_{0} G_{\lambda}$, with $\phi_{0 \lambda} \in \mathcal{S}\left(\mathbb{R}^{3}\right)$, then the moment of inertia of the solution $\psi(t)$ associated to $\psi_{0}$, is an element of $C^{2}\left(0, T^{*}\right)$ and

$$
\ddot{I}(t)=8 E\left(\psi_{0}\right)+4 \gamma \frac{\sigma-1}{\sigma+1}|q(t)|^{2 \sigma+2} .
$$

Proof. As a first step, we shall compute the second derivative of the regularized moment of inertia $I_{\varepsilon}(t)$, with $t \in\left[0, T^{*}\right)$. Let us choose $T$ such that $t<T<T^{*}$.

From Proposition 2.10 and applying the dominated convergence theorem, one concludes

$$
\begin{aligned}
\ddot{I}_{\varepsilon}(t) & =\frac{1}{2 \pi^{3}} \operatorname{Im} \int_{\mathbb{R}^{3}} \mathrm{~d} \mathbf{k} \mathrm{e}^{-|\mathbf{k}|^{2} \varepsilon} \mathbf{k} \cdot\left[\partial_{t} \hat{\psi}(t, \mathbf{k}) \nabla \overline{\hat{\psi}(t, \mathbf{k})}+\hat{\psi}(t, \mathbf{k}) \partial_{t} \nabla \overline{\hat{\psi}(t, \mathbf{k})}\right] \\
& =\frac{1}{2 \pi^{3}} \operatorname{Re} q(t) \int_{\mathbb{R}^{3}} \mathrm{~d} \mathbf{k} \mathrm{e}^{-|\mathbf{k}|^{2} \varepsilon} \mathbf{k} \cdot \nabla \overline{\hat{\psi}(t, \mathbf{k})}+\frac{1}{\pi^{3}} \int_{\mathbb{R}^{3}} \mathrm{dk}^{-|\mathbf{k}|^{2} \varepsilon}|\mathbf{k}|^{2}|\psi(t, \mathbf{k})|^{2} .
\end{aligned}
$$

Let us analyze the first term in the r.h.s. of (3.3). Using the divergence theorem we obtain

$$
\int_{\mathbb{R}^{3}} \mathrm{~d} \mathbf{k} \mathrm{e}^{-|\mathbf{k}|^{2} \varepsilon} \mathbf{k} \cdot \nabla \overline{\hat{\psi}(t, \mathbf{k})}=\int_{\mathbb{R}^{3}} \mathrm{~d} \mathbf{k} \mathrm{e}^{-|\mathbf{k}|^{2} \varepsilon}\left(-3+2|\mathbf{k}|^{2} \varepsilon\right) \overline{\hat{\psi}(t, \mathbf{k})}
$$


while for the second term we have

$$
\begin{aligned}
& \int_{\mathbb{R}^{3}} \mathrm{~d} \mathbf{k} \mathrm{e}^{-|\mathbf{k}|^{2} \varepsilon}|\mathbf{k}|^{2}|\hat{\psi}(t, \mathbf{k})|^{2} \\
& \quad=\int_{\mathbb{R}^{3}} \mathrm{~d} \mathbf{k} \mathrm{e}^{-|\mathbf{k}|^{2} \varepsilon}|\mathbf{k}|^{2}|\hat{\phi}(t, \mathbf{k})|^{2}+2 \operatorname{Re} q(t) \int_{\mathbb{R}^{3}} \mathrm{~d} \mathbf{k} \mathrm{e}^{-|\mathbf{k}|^{2} \varepsilon} \overline{\hat{\psi}(t, \mathbf{k})}-|q(t)|^{2} \int_{\mathbb{R}^{3}} \mathrm{~d} \mathbf{k} \frac{\mathrm{e}^{-|\mathbf{k}|^{2} \varepsilon}}{|\mathbf{k}|^{2}} .
\end{aligned}
$$

Using Eqs. (3.4), (3.5) in Eq. (3.3) we have

$$
\begin{aligned}
\ddot{I}_{\varepsilon}(t)= & \frac{1}{\pi^{3}} \int_{\mathbb{R}^{3}} \mathrm{~d} \mathbf{k} \mathrm{e}^{-|\mathbf{k}|^{2} \varepsilon}|\mathbf{k}|^{2}|\hat{\phi}(t, \mathbf{k})|^{2}+\frac{1}{2 \pi^{3}} \operatorname{Re} q(t) \int_{\mathbb{R}^{3}} \mathrm{~d} \mathbf{k} \mathrm{e}^{-|\mathbf{k}|^{2} \varepsilon} \overline{\hat{\psi}(t, \mathbf{k})} \\
& +\frac{\varepsilon}{\pi^{3}} \operatorname{Re} \int_{\mathbb{R}^{3}} \mathrm{dk} \mathrm{e}^{-|\mathbf{k}|^{2} \varepsilon}|\mathbf{k}|^{2} \overline{\hat{\psi}(t, \mathbf{k})}-\frac{2}{\pi^{3 / 2} \sqrt{\varepsilon}}|q(t)|^{2} .
\end{aligned}
$$

An explicit computation gives

$$
\begin{aligned}
& \int_{\mathbb{R}^{3}} \mathrm{~d} \mathbf{k} \hat{\psi}(t, \mathbf{k}) \mathrm{e}^{-|\mathbf{k}|^{2} \varepsilon}=J_{\varepsilon}(t)+2 \pi^{3 / 2} \frac{q(t)}{\sqrt{\varepsilon}}, \\
& \int_{\mathbb{R}^{3}} \mathrm{~d} \mathbf{k} \hat{\psi}(t, \mathbf{k})|\mathbf{k}|^{2} \mathrm{e}^{-|\mathbf{k}|^{2} \varepsilon}=\mathrm{i} \frac{\mathrm{d}}{\mathrm{d} t} J_{\varepsilon}(t)+2 \mathrm{i} \pi^{3 / 2} \frac{\dot{q}(t)}{\sqrt{\varepsilon}}+\frac{\pi^{3 / 2}}{\varepsilon^{3 / 2}} q(t),
\end{aligned}
$$

where

$$
J_{\varepsilon}(t)=8 \pi^{3}\left(U(t) \psi_{0 \varepsilon}\right)(0)-2 \pi^{3 / 2} \sqrt{-\mathrm{i}} \int_{0}^{t} \mathrm{~d} s \frac{\dot{q}(s)}{\sqrt{t-s-\mathrm{i} \varepsilon}}-2 \pi^{3 / 2} \sqrt{-\mathrm{i}} \frac{q_{0}}{\sqrt{t-\mathrm{i} \varepsilon}}
$$

and

$$
\psi_{0 \varepsilon}(\mathbf{x})=(2 \pi)^{-3} \int_{\mathbb{R}^{3}} \mathrm{~d} \mathbf{k} \hat{\psi}_{0}(\mathbf{k}) \mathrm{e}^{-|\mathbf{k}|^{2} \varepsilon} \mathrm{e}^{-\mathrm{i} \mathbf{k} \cdot \mathbf{x}} .
$$

From (3.6), (3.7) and (3.8) we have

$$
\begin{aligned}
\ddot{I}_{\varepsilon}(t)= & \frac{1}{\pi^{3}} \int_{\mathbb{R}^{3}} \mathrm{~d} \mathbf{k}|\mathbf{k}|^{2}|\hat{\phi}(t, \mathbf{k})|^{2} \mathrm{e}^{-|\mathbf{k}|^{2} \varepsilon}+\frac{1}{2 \pi^{3}} \operatorname{Re}\left[q(t) \overline{J_{\varepsilon}(t)}\right] \\
& -2 \frac{\sqrt{\varepsilon}}{\pi^{3 / 2}} \operatorname{Im}[\overline{q(t)} \dot{q}(t)]-\frac{\varepsilon}{\pi^{3}} \operatorname{Im}\left[\overline{q(t)} \frac{\mathrm{d} J_{\varepsilon}}{\mathrm{d} t}(t)\right] \\
\equiv & (\mathrm{I})+(\mathrm{II})+(\mathrm{III})+(\mathrm{IV}) .
\end{aligned}
$$

In order to estimate the function $J_{\varepsilon}$, let us recall that, for any $\lambda>0$

$$
\begin{aligned}
8 \pi^{3}\left[U(t) \psi_{0}\right](0) & =\int_{\mathbb{R}^{3}} \mathrm{~d} \mathbf{k} \mathrm{e}^{-\mathrm{i}|\mathbf{k}|^{2} t} \mathrm{e}^{-|\mathbf{k}|^{2} \varepsilon}\left[\hat{\phi}_{0 \lambda}(\mathbf{k})+\frac{q_{0}}{|\mathbf{k}|^{2}+\lambda}\right] \\
& =\int_{\mathbb{R}^{3}} \mathrm{~d} \mathbf{k} \mathrm{e}^{-i|\mathbf{k}|^{2} t} \mathrm{e}^{-|\mathbf{k}|^{2} \varepsilon} \hat{\phi}_{0 \lambda}(\mathbf{k})+\frac{2 \pi^{3 / 2} q_{0}}{\sqrt{\varepsilon+\mathrm{i} t}}-4 \pi \lambda q_{0} \int_{0}^{\infty} \mathrm{d} k \frac{\mathrm{e}^{-\mathrm{i} k^{2} t} \mathrm{e}^{-k^{2} \varepsilon}}{k^{2}+\lambda} .
\end{aligned}
$$


Therefore

$$
8 \pi^{3}\left|\left[U(t) \psi_{0}\right](0)\right| \leqslant\left\|\hat{\phi}_{0 \lambda}\right\|_{L^{1}\left(\mathbb{R}^{3}\right)}+\frac{2 \pi^{3 / 2}\left|q_{0}\right|}{\left(\varepsilon^{2}+t^{2}\right)^{1 / 4}}+2 \pi^{2} \sqrt{\lambda}\left|q_{0}\right| .
$$

From (3.14) one easily finds the following estimates

$$
\begin{aligned}
& 8 \pi^{3}\left|\left[U(t) \psi_{0}\right](0)\right| \leqslant\left\|\hat{\phi}_{0 \lambda}\right\|_{L^{1}\left(\mathbb{R}^{3}\right)}+2 \pi^{2} \sqrt{\lambda}\left|q_{0}\right|+2 \pi^{3 / 2} \frac{\left|q_{0}\right|}{\sqrt{t}} \\
& 8 \pi^{3}\left|\left[U(t) \psi_{0}\right](0)\right| \leqslant\left\|\hat{\phi}_{0 \lambda}\right\|_{L^{1}\left(\mathbb{R}^{3}\right)}+2 \pi^{2} \sqrt{\lambda}\left|q_{0}\right|+2 \pi^{3 / 2} \frac{\left|q_{0}\right|}{\sqrt{\varepsilon}} .
\end{aligned}
$$

Recalling definition (3.9), from (3.15) and (3.16) one finally has

$$
\begin{aligned}
& \left|J_{\varepsilon}(t)\right| \leqslant\left\|\hat{\phi}_{0 \lambda}\right\|_{L^{1}\left(\mathbb{R}^{3}\right)}+2 \pi^{2} \sqrt{\lambda}\left|q_{0}\right|+4 \pi^{3 / 2} \frac{\left|q_{0}\right|}{\sqrt{t}}+2 \pi^{3 / 2} \int_{0}^{t} \mathrm{~d} s \frac{|\dot{q}(s)|}{\sqrt{t-s}} \equiv h_{1}(t), \\
& \left|J_{\varepsilon}(t)\right| \leqslant\left\|\hat{\phi}_{0 \lambda}\right\|_{L^{1}\left(\mathbb{R}^{3}\right)}+2 \pi^{2} \sqrt{\lambda}\left|q_{0}\right|+\frac{2 \pi^{3 / 2}}{\sqrt{\varepsilon}}\left(2\left|q_{0}\right|+\|\dot{q}\|_{L^{1}(0, T)}\right) \equiv h_{2}(t) .
\end{aligned}
$$

We note that the function $h_{1}$ defined in (3.17) is integrable in $(0, T)$.

Let us consider the equality

$$
I_{\varepsilon}(t)=I_{\varepsilon}(0)+\dot{I}_{\varepsilon}(0) t+\int_{0}^{t} \mathrm{~d} s \int_{0}^{s} \mathrm{~d} s^{\prime} \ddot{I}_{\varepsilon}\left(s^{\prime}\right),
$$

where, following Theorem 4

$$
\dot{I}_{\varepsilon}(0)=\frac{1}{2 \pi^{3}} \operatorname{Im} \int_{\mathbb{R}^{3}} \mathrm{~d} \mathbf{k} \hat{\psi}_{0}(\mathbf{k}) \mathbf{k} \cdot \overline{\nabla_{\mathbf{k}} \hat{\psi}_{0}(\mathbf{k})} \mathrm{e}^{-|\mathbf{k}|^{2} \varepsilon} .
$$

We want to take the limit $\varepsilon \rightarrow 0$ in Eq. (3.19). The only delicate term is the last one, where $\ddot{I}_{\varepsilon}$ is given by (3.12).

Applying the monotone convergence theorem, one obtains

$$
\lim _{\varepsilon \rightarrow 0}(\mathrm{I})=8\left\|\nabla \phi\left(s^{\prime}\right)\right\|_{L^{2}\left(\mathbb{R}^{3}\right)} \cdot
$$

Concerning (II), we have

$$
\lim _{\varepsilon \rightarrow 0}(\mathrm{II})=\frac{1}{2 \pi^{2}} \lim _{\varepsilon \rightarrow 0} \int_{0}^{t} \mathrm{~d} s \int_{0}^{s} \mathrm{~d} s^{\prime} \operatorname{Re}\left[\overline{q\left(s^{\prime}\right)} J_{\varepsilon}\left(s^{\prime}\right)\right] .
$$

From estimate (3.17)

$$
\left|q\left(s^{\prime}\right) J_{\varepsilon}\left(s^{\prime}\right)\right| \leqslant\|q\|_{L^{\infty}(0, T)} h_{1}\left(s^{\prime}\right)
$$

and by dominated convergence theorem we obtain

$$
\lim _{\varepsilon \rightarrow 0}(\mathrm{II})=\frac{1}{2 \pi^{2}} \int_{0}^{t} \mathrm{~d} s \int_{0}^{s} \mathrm{~d} s^{\prime} \operatorname{Re}\left[\overline{q\left(s^{\prime}\right)} J_{0}\left(s^{\prime}\right)\right] .
$$


Applying the operator $D$, defined by $(D g)(t)=\frac{\mathrm{d}}{\mathrm{d} t} \int_{0}^{t} \mathrm{~d} s \frac{g(s)}{\sqrt{t-s}}$, to both sides of Eq. (1.9), we get

$$
\int_{0}^{t} \mathrm{~d} s \frac{\dot{q}(s)}{\sqrt{t-s}}+\frac{q_{0}}{\sqrt{t}}-4 \sqrt{\mathrm{i}} \pi^{3 / 2}\left(U_{3}(t) \psi_{0}\right)(0)=-4 \sqrt{\mathrm{i}} \pi^{3 / 2} \gamma|q(t)|^{2 \sigma} q(t)
$$

and hence

$$
J_{0}(t)=8 \pi^{3} \gamma|q(t)|^{2 \sigma} q(t),
$$

thus

$$
\lim _{\varepsilon \rightarrow 0}(\mathrm{II})=4 \gamma \int_{0}^{t} \mathrm{~d} s \int_{0}^{s} \mathrm{~d} s^{\prime}\left|q\left(s^{\prime}\right)\right|^{2 \sigma+2} .
$$

It is easily seen that $\lim _{\varepsilon \rightarrow 0}(\mathrm{III})=0$, so we just compute $\lim _{\varepsilon \rightarrow 0}(\mathrm{IV})$.

Integrating by parts, we obtain

$$
\begin{aligned}
(\mathrm{IV}) & =\frac{\varepsilon}{\pi^{3}} \int_{0}^{t} \mathrm{~d} s \operatorname{Im}\left[\overline{q(s)} J_{\varepsilon}(s)\right]-\frac{\varepsilon}{\pi^{3}} t \operatorname{Im}\left[\overline{q_{0}} J_{\varepsilon}(0)\right]-\frac{\varepsilon}{\pi^{3}} \int_{0}^{t} \mathrm{~d} s \int_{0}^{t} \mathrm{~d} s^{\prime} \operatorname{Im}\left[\overline{\dot{q}\left(s^{\prime}\right)} J_{\varepsilon}\left(s^{\prime}\right)\right] \\
& \equiv(\mathrm{IVa})+(\mathrm{IVb})+(\mathrm{IV} \mathrm{c}) .
\end{aligned}
$$

Using estimate (3.23) and applying the dominated convergence theorem, one finds $\lim _{\varepsilon \rightarrow 0}(\mathrm{IVa})=0$. Moreover from estimate (3.18)

$$
\begin{aligned}
& (\mathrm{IVb}) \leqslant t \frac{\left|q_{0}\right|}{\pi^{3}} \varepsilon h_{2}(\varepsilon), \\
& (\mathrm{IVc}) \leqslant t \frac{\left\|q_{0}\right\|_{L^{1}(0, T)}}{\pi^{3}} \varepsilon h_{2}(\varepsilon) .
\end{aligned}
$$

From (3.21), (3.27) and the definition of the energy we have

$$
\lim _{\varepsilon \rightarrow 0} \int_{0}^{t} \mathrm{~d} s \int_{0}^{s} \mathrm{~d} s^{\prime} \ddot{I}_{\varepsilon}\left(s^{\prime}\right)=4 t^{2} E\left(\psi_{0}\right)+4 \gamma \frac{\sigma-1}{\sigma+1} \int_{0}^{t} \mathrm{~d} s \int_{0}^{s} \mathrm{~d} s^{\prime}\left|q\left(s^{\prime}\right)\right|^{2 \sigma+2}
$$

and then from (3.19) we conclude

$$
\ddot{I}(t)=8 E\left(\psi_{0}\right)+4 \gamma \frac{\sigma-1}{\sigma+1}|q(t)|^{2 \sigma+2} .
$$

This concludes the proof of Proposition 5.

In order to extend the above result to an arbitrary element in the space of finite energy $V$, we introduce the following sequence of $\delta$-approximating regular functions:

$$
\delta^{(m)}(\mathbf{x})=\left(\frac{m}{\pi}\right)^{3 / 2} \mathrm{e}^{-m|\mathbf{x}|^{2}}
$$

and denote

$$
g^{(m)}(\mathbf{k})=\hat{\delta}^{(m)}(\mathbf{k})=\mathrm{e}^{-|\mathbf{k}|^{2} /(4 m)} .
$$

For any $\phi_{0 \lambda} \in H^{1}\left(\mathbb{R}^{3}\right)$ we consider the following approximating sequence:

$$
\phi_{0 \lambda}^{(m)}=\frac{1}{2} \delta^{(m)} *\left(g^{(m)} \phi_{0 \lambda}\right)+\frac{1}{2} g^{(m)}\left(\delta^{(m)} * \phi_{0 \lambda}\right)
$$


which takes the same form in Fourier space:

$$
\hat{\phi}_{0 \lambda}^{(m)}=\frac{1}{2} g^{(m)}\left(\delta^{(m)} * \hat{\phi}_{0 \lambda}\right)+\frac{1}{2} \delta^{(m)} *\left(g^{(m)} \hat{\phi}_{0 \lambda}\right) .
$$

It is immediately seen that $\phi_{0 \lambda}^{(m)}$ belongs to the Schwarz space, $\phi_{0 \lambda}^{(m)} \rightarrow \phi_{0 \lambda}$ in $H^{1}\left(\mathbb{R}^{3}\right)$ and $|\mathbf{x}| \phi_{0 \lambda}^{(m)} \rightarrow|\mathbf{x}| \phi_{0 \lambda}$ in $L^{2}\left(\mathbb{R}^{3}\right)$; moreover, from [1], Theorem 8, we know that $\phi_{\lambda}^{(m)}(t) \rightarrow \phi_{\lambda}(t)$ in $H^{1}\left(\mathbb{R}^{3}\right)$ and $|\mathbf{x}| \phi_{\lambda}^{(m)}(t) \rightarrow|\mathbf{x}| \phi_{\lambda}(t)$ in $L^{2}\left(\mathbb{R}^{3}\right)$, which implies $|\mathbf{x}| \psi_{\lambda}^{(m)}(t) \rightarrow|\mathbf{x}| \psi_{\lambda}(t)$ in $L^{2}\left(\mathbb{R}^{3}\right)$. Using the above approximating procedure we can prove the following

Theorem 6. For any initial datum $\psi_{0}=\phi_{0 \lambda}+q_{0} G_{\lambda}$, with $\phi_{0 \lambda} \in H^{1}\left(\mathbb{R}^{3}\right)$, the moment of inertia of the solution $\psi(t)$ associated to $\psi_{0}$, is an element of $C^{2}\left(0, T^{*}\right)$ and

$$
\ddot{I}(t)=8 E\left(\psi_{0}\right)+4 \gamma \frac{\sigma-1}{\sigma+1}|q(t)|^{2 \sigma+2} .
$$

Proof. Let $\phi_{0 \lambda}^{(m)}$ be the sequence defined in (3.35). Let us denote $\psi^{(m)}(t)=\phi_{\lambda}^{(m)}(t)+q^{(m)}(t) G_{\lambda}$ the solution associated to the initial datum $\psi_{0}^{(m)}=\phi_{0 \lambda}^{(m)}+q_{0} G_{\lambda}$, and let $I^{(m)}(t)$ be the corresponding moment of inertia.

We first prove that $I^{(m)}(0)$ and $\dot{I}^{(m)}(0)$ converge to $I(0)$ and $\dot{I}(0)$ respectively.

Indeed

$$
\begin{aligned}
\left|I^{(m)}(0)-I(0)\right| \leqslant & \left|\left\||\mathbf{x}| \phi_{0 \lambda}^{(m)}\right\|_{L^{2}\left(\mathbb{R}^{3}\right)}^{2}-\left\||\mathbf{x}| \phi_{0 \lambda}\right\|_{L^{2}\left(\mathbb{R}^{3}\right)}^{2}\right| \\
& +\frac{1}{2 \pi^{3}}\left|q_{0}\right|\left|\int_{\mathbb{R}^{3}} \mathrm{~d} \mathbf{k}\left(\nabla_{\mathbf{k}} \hat{\phi}_{0 \lambda}^{(m)}(\mathbf{k})-\nabla_{\mathbf{k}} \hat{\phi}_{0 \lambda}(\mathbf{k})\right) \cdot \frac{\mathbf{k}}{\left(|\mathbf{k}|^{2}+\lambda\right)^{2}}\right| .
\end{aligned}
$$

Due to the convergence of the approximating sequence and the Cauchy-Schwarz inequality we have $I^{(m)}(0) \rightarrow$ $I(0)$ for $m \rightarrow \infty$.

Concerning $\dot{I}^{(m)}(0)$, from Formula (2.10), we have

$$
\begin{aligned}
\left|\dot{I}^{(m)}(0)-\dot{I}(0)\right| \leqslant & \frac{1}{2 \pi^{3}}\left|\int_{\mathbb{R}^{3}} \mathrm{~d} \mathbf{k} \hat{\phi}_{0 \lambda}^{(m)}(\mathbf{k}) \mathbf{k} \cdot \overline{\nabla_{\mathbf{k}} \hat{\phi}_{0 \lambda}^{(m)}(\mathbf{k})}-\int_{\mathbb{R}^{3}} \mathrm{~d} \mathbf{k} \hat{\phi}_{0 \lambda}(\mathbf{k}) \mathbf{k} \cdot \overline{\nabla_{\mathbf{k}} \hat{\phi}_{0 \lambda}(\mathbf{k})}\right| \\
& +\frac{\left|q_{0}\right|}{2 \pi^{3}}\left|\int_{\mathbb{R}^{3}} \mathrm{~d} \mathbf{k} \frac{\mathbf{k}}{|\mathbf{k}|^{2}+\lambda} \cdot\left[\nabla_{\mathbf{k}} \hat{\phi}_{0 \lambda}^{(m)}(\mathbf{k})-\nabla_{\mathbf{k}} \hat{\phi}_{0 \lambda}(\mathbf{k})\right]\right| \\
& +\frac{\left|q_{0}\right|}{\pi^{3}}\left|\int_{\mathbb{R}^{3}} \mathrm{~d} \mathbf{k} \frac{|\mathbf{k}|^{2}}{\left(|\mathbf{k}|^{2}+\lambda\right)^{2}}\left[\hat{\phi}_{0 \lambda}^{(m)}(\mathbf{k})-\hat{\phi}_{0 \lambda}(\mathbf{k})\right]\right| \\
= & (\mathrm{V})+(\mathrm{VI})+(\mathrm{VII}) .
\end{aligned}
$$

From the convergence of the approximating sequence it follows

$$
(\mathrm{V}) \leqslant 4\left|\left(\nabla \phi_{0 \lambda}^{(m)}, \mathbf{x} \phi_{0 \lambda}^{(m)}\right)_{L^{2}\left(\mathbb{R}^{3}\right)}-\left(\nabla \phi_{0 \lambda}, \mathbf{x} \phi_{0 \lambda}\right)_{L^{2}\left(\mathbb{R}^{3}\right)}\right| \rightarrow 0 \quad \text { for } m \rightarrow \infty .
$$

Moreover integrating by parts one obtains

$$
\begin{aligned}
(\mathrm{VI}) & \leqslant \frac{\left|q_{0}\right|}{2 \pi^{3}}\left|\int_{\mathbb{R}^{3}} \mathrm{~d} \mathbf{k} \frac{|\mathbf{k}|^{2}+3 \lambda}{\left(|\mathbf{k}|^{2}+\lambda\right)^{2}}\right|\left(\hat{\phi}_{0 \lambda}^{(m)}(\mathbf{k})-\hat{\phi}_{0 \lambda}(\mathbf{k})\right) \\
& \leqslant 4\left|q_{0}\right| M_{1}\left\|\nabla \phi_{0 \lambda}^{(m)}-\nabla \phi_{0 \lambda}\right\|_{L^{2}\left(\mathbb{R}^{3}\right)},
\end{aligned}
$$


where we applied the Cauchy-Schwarz inequality and we defined

$$
M_{1}^{2} \equiv \int_{\mathbb{R}^{3}} \mathrm{~d} \mathbf{k} \frac{\left(|\mathbf{k}|^{2}+3 \lambda\right)^{2}}{|\mathbf{k}|^{2}\left(|\mathbf{k}|^{2}+\lambda\right)^{4}}
$$

For the last term in (3.40) we have

$$
(\mathrm{VII}) \leqslant 8\left|q_{0}\right| M_{2}\left\|\phi_{0 \lambda}^{(m)}-\phi_{0 \lambda}\right\|_{L^{2}\left(\mathbb{R}^{3}\right)},
$$

where we applied the Cauchy-Schwarz inequality and we denoted

$$
M_{2}^{2} \equiv \int_{\mathbb{R}^{3}} \mathrm{~d} \mathbf{k} \frac{|\mathbf{k}|^{4}}{\left(|\mathbf{k}|^{2}+\lambda\right)^{4}} .
$$

Taking the limit $m \rightarrow \infty$ in both sides of the equality

$$
I^{(m)}(t)=I^{(m)}(0)+t \dot{I}^{(m)}(0)+4 t^{2} E\left(\psi_{0}^{(m)}\right)+4 \gamma \frac{\sigma-1}{\sigma+1} \int_{0}^{t} \mathrm{~d} s \int_{0}^{s} \mathrm{~d} s^{\prime}\left|q^{(m)}\left(s^{\prime}\right)\right|^{2 \sigma+2}
$$

and using (3.41), (3.42), (3.44), we obtain

$$
I(t)=I(0)+t \dot{I}(0)+4 t^{2} \lim _{m \rightarrow \infty} E\left(\psi_{0}^{(m)}\right)+4 \gamma \frac{\sigma-1}{\sigma+1} \lim _{m \rightarrow \infty} \int_{0}^{t} \mathrm{~d} s \int_{0}^{s} \mathrm{~d} s^{\prime}\left|q^{(m)}\left(s^{\prime}\right)\right|^{2 \sigma+2} .
$$

Since the energy functional is continuous in $V$ and $q^{(m)}$ converges to $q$ in $H^{3 / 4}(0, T)$ and therefore in $C^{0}(0, T)$ (see [1]), we finally obtain

$$
I(t)=I(0)+t \dot{I}(0)+4 t^{2} E\left(\psi_{0}\right)+4 \gamma \frac{\sigma-1}{\sigma+1} \int_{0}^{t} \mathrm{~d} s \int_{0}^{s} \mathrm{~d} s^{\prime}\left|q\left(s^{\prime}\right)\right|^{2 \sigma+2}
$$

which implies

$$
\ddot{I}(t)=8 E\left(\psi_{0}\right)+4 \gamma \frac{\sigma-1}{\sigma+1}|q(t)|^{2 \sigma+2} .
$$

This concludes the proof of Theorem 6 .

\section{Sufficient condition for the blow-up}

Using Theorem 6 and a modified version of the uncertainty principle, we can now state the result on the existence of blow-up solutions.

Theorem 7. Let $\gamma<0, \sigma \geqslant 1$ and $\psi_{0}=\phi_{0 \lambda}+q_{0} G_{\lambda}$, with $\phi_{0 \lambda} \in H^{1}\left(\mathbb{R}^{3}\right),|\mathbf{x}| \psi_{0} \in L^{2}\left(\mathbb{R}^{3}\right), E\left(\psi_{0}\right)<0$.

Then the corresponding solution $\psi(t)$ is a blow-up solution for both $t>0$ and $t<0$.

Proof. Using the identity

$$
\|g\|_{L^{2}\left(\mathbb{R}^{3}\right)}^{2}=-\frac{2}{3} \operatorname{Re} \int_{\mathbb{R}^{3}} \mathrm{~d} \mathbf{x} \overline{g(\mathbf{x})} \mathbf{x} \cdot \nabla g(\mathbf{x})
$$


for $g \in L^{2}\left(\mathbb{R}^{3}\right), \bar{g} \mathbf{x} \cdot \nabla g \in L^{1}\left(\mathbb{R}^{3}\right)$, the conservation of the $L^{2}$-norm and Schwartz inequality we have

$$
\begin{aligned}
\left\|\psi_{0}\right\|_{L^{2}\left(\mathbb{R}^{3}\right)}^{2} & =\|\psi(t)\|_{L^{2}\left(\mathbb{R}^{3}\right)}^{2} \\
& \leqslant \frac{2}{3}\left|\int_{\mathbb{R}^{3}} \mathrm{~d} \mathbf{x} \overline{\psi(t, \mathbf{x})} \mathbf{x} \cdot \nabla \phi_{\lambda}(t, \mathbf{x})\right|+\frac{2}{3}|q(t)|\left|\int_{\mathbb{R}^{3}} \mathrm{~d} \mathbf{x} \overline{\psi(t, \mathbf{x})} \mathbf{x} \cdot \nabla G_{\lambda}(\mathbf{x})\right| \\
& \leqslant \frac{2}{3}(I(t))^{1 / 2}\left\|\nabla \phi_{\lambda}(t)\right\|_{L^{2}\left(\mathbb{R}^{3}\right)}+\frac{2}{3}|q(t)|\|\psi(t)\|_{L^{2}\left(\mathbb{R}^{3}\right)}\left\|\mathbf{x} \cdot \nabla G_{\lambda}\right\|_{L^{2}\left(\mathbb{R}^{3}\right)} \\
& =\frac{2}{3}(I(t))^{1 / 2}\left\|\nabla \phi_{\lambda}(t)\right\|_{L^{2}\left(\mathbb{R}^{3}\right)}+\frac{4 \sqrt{\pi}}{3 \lambda^{1 / 4}}|q(t)|\left\|\psi_{0}\right\|_{L^{2}\left(\mathbb{R}^{3}\right)}\left(\int_{0}^{\infty} \mathrm{d} r(1+r)^{2} \mathrm{e}^{-2 r}\right)^{1 / 2} \\
& \equiv \frac{2}{3}(I(t))^{1 / 2}\left\|\nabla \phi_{\lambda}(t)\right\|_{L^{2}\left(\mathbb{R}^{3}\right)}+\frac{2 \sqrt{5 \pi}}{3 \lambda^{1 / 4}}|q(t)|\left\|\psi_{0}\right\|_{L^{2}\left(\mathbb{R}^{3}\right)} .
\end{aligned}
$$

From the definition of $\phi_{\lambda}$ and $\phi$, one has

$$
\nabla \phi_{\lambda}(t, \mathbf{x})=\nabla \phi(t, \mathbf{x})+q(t) \frac{1+\mathrm{e}^{-\sqrt{\lambda}|\mathbf{x}|}(\sqrt{\lambda}|\mathbf{x}|-1)}{4 \pi|\mathbf{x}|^{2}} .
$$

Therefore

$$
\left\|\nabla \phi_{\lambda}(t)\right\|_{L^{2}\left(\mathbb{R}^{3}\right)}^{2} \leqslant 2\|\nabla \phi(t)\|_{L^{2}\left(\mathbb{R}^{3}\right)}^{2}+2 \sqrt{\lambda} M_{3}|q(t)|^{2},
$$

where

$$
M_{3} \equiv \frac{1}{4 \pi} \int_{0}^{\infty} \frac{\mathrm{d} \eta}{\eta^{2}}\left[1+\mathrm{e}^{-\sqrt{\lambda} \eta}(\eta-1)\right]^{2} .
$$

From (4.2) and the definition of the energy

$$
\left\|\psi_{0}\right\|_{L^{2}\left(\mathbb{R}^{3}\right)}^{4} \leqslant \frac{16}{9} I(t)\left[E\left(\psi_{0}\right)+\frac{|\gamma|}{\sigma+1}|q(t)|^{2 \sigma+2}+\sqrt{\lambda} M_{3}|q(t)|^{2}\right]+\frac{40 \pi}{9 \sqrt{\lambda}}\left\|\psi_{0}\right\|_{L^{2}\left(\mathbb{R}^{3}\right)}^{2} .
$$

Let us consider the evolution for $t>0$.

From Theorem 6 we know that either the solution ceases to exist at a time $T^{*}$ such that $\sup _{t \in\left[0, T^{*}\right)}|I(t)|>0$ or there exists $T_{c}<\infty$ such that $\lim _{t \rightarrow T_{c}} I(t)=0$.

In the former case the blow-up alternative guarantees the occurrence of the blow-up. In the latter case assume that there is no blow-up in $\left[0, T_{c}\right]$, i.e., $|q(t)|$ remains bounded in $\left[0, T_{c}\right]$. Then, given $\lambda>0$, from (4.6), we have:

$$
\left\|\psi_{0}\right\|_{L^{2}\left(\mathbb{R}^{3}\right)}^{2} \leqslant \frac{2 \sqrt{5 \pi}}{3 \lambda^{1 / 4}}\left\|\psi_{0}\right\|_{L^{2}\left(\mathbb{R}^{3}\right)}\|q\|_{L^{\infty}\left(0, T_{C}\right)} .
$$

The r.h.s. of (4.7) can be made arbitrarily small choosing $\lambda$ suitably large, so we get a contradiction. Therefore the solution blows up in $\left[0, T_{c}\right]$ and in particular $T_{c}=T^{*}$.

The same argument can be repeated for the backward problem and this concludes the proof of Theorem 7.

\section{Additional symmetries in the critical case $\sigma=1$}

From Theorem 7 and from [1], Theorem 12, we know that $\sigma=1$ is the lowest power for which the system exhibits blow-up solutions. 
For the convenience of the reader we rewrite Eq. (1.9) for $\sigma=1$

$$
q(t)+4 \sqrt{\pi \mathrm{i}} \gamma \int_{0}^{t} \mathrm{~d} s \frac{|q(s)|^{2} q(s)}{\sqrt{t-s}}=4 \sqrt{\pi \mathrm{i}} \int_{0}^{t} \mathrm{~d} s \frac{\left[U(s) \psi_{0}\right](0)}{\sqrt{t-s}} .
$$

Following the literature on the standard nonlinear Schrödinger equation (see, e.g., [5,6] and references therein), we shall call the case with $\sigma=1$ the critical case for the Schrödinger equation with a nonlinearity concentrated in a point.

In this section we shall go deeper in the analogy with the standard NLSE showing that additional symmetries are shared by the critical cases.

In particular we show that the system is endowed with the dilation and gauge symmetries, which, put together, give the pseudo-conformal invariance law.

Proposition 8. Let us consider $\psi(t)$, solution to the problem (1.5), (5.1), associated to the initial datum $\psi_{0}$ in the domain of finite energy $V$.

Then,

(1) $\psi^{\varepsilon}(t, \mathbf{x})=\varepsilon^{3 / 2} \psi\left(\varepsilon^{2} t, \varepsilon \mathbf{x}\right)$ is the solution associated to the initial datum $\psi_{0}^{\varepsilon}(\mathbf{x})=\varepsilon^{3 / 2} \psi_{0}(\varepsilon \mathbf{x})$.

(2) $\psi^{T}(t, \mathbf{x})=\left(\frac{T}{T+t}\right)^{3 / 2} \mathrm{e}^{\mathrm{i} \frac{|\mathbf{x}|^{2}}{4(t+T)}} \psi\left(\frac{t T}{t+T}, \frac{\mathbf{x} T}{t+T}\right)$ is the solution associated to the inital datum $\psi_{0}^{T}(\mathbf{x})=\mathrm{e}^{\mathrm{i} \frac{|\mathbf{x}|^{2}}{4 T}} \psi_{0}(\mathbf{x})$.

Proof. We start with the dilation symmetry. We first recall that the free Schrödinger evolution is endowed with such a symmetry. Then, we only discuss the second term in (1.5).

After rescaling the variable $s$, it is immediately seen that

$$
\int_{0}^{t} \mathrm{~d} s \frac{\left[U(s) \psi_{0}^{\varepsilon}\right](0)}{\sqrt{t-s}}=\sqrt{\varepsilon} \int_{0}^{\varepsilon^{2} t} \mathrm{~d} s \frac{\left[U(s) \psi_{0}\right](0)}{\sqrt{\varepsilon^{2} t-s}} .
$$

Therefore from (5.1) we have

$$
\sqrt{\varepsilon} q\left(\varepsilon^{2} t\right)+4 \sqrt{\varepsilon \pi \mathrm{i}} \gamma \int_{0}^{\varepsilon^{2} t} \mathrm{~d} s \frac{|q(s)|^{2} q(s)}{\sqrt{\varepsilon^{2} t-s}}=4 \sqrt{\pi \mathrm{i}} \int_{0}^{t} \mathrm{~d} s \frac{\left[U(s) \psi_{0}^{\varepsilon}\right](0)}{\sqrt{t-s}},
$$

where $q$ solves (5.1) for the initial datum $\psi_{0}$. We now define $q^{\varepsilon}(t) \equiv \sqrt{\varepsilon} q\left(\varepsilon^{2} t\right)$ and, after rescaling the variable $s$ in the integral term in the 1.h.s. of (5.3), we find that $q^{\varepsilon}$ solves (5.1) for the initial datum $\psi_{0}^{\varepsilon}$.

Recalling that

$$
U\left(t-\frac{s^{\prime}}{\varepsilon^{2}} ; \mathbf{x}\right)=\varepsilon^{3} U\left(\varepsilon^{2} t-s^{\prime} ; \varepsilon \mathbf{x}\right)
$$

we conclude

$$
\int_{0}^{t} \mathrm{~d} s U(t-s ; \mathbf{x}) q^{\varepsilon}(s)=\varepsilon^{3 / 2} \int_{0}^{\varepsilon^{2} t} \mathrm{~d} s^{\prime} U\left(\varepsilon^{2} t-s^{\prime} ; \varepsilon \mathbf{x}\right) q\left(s^{\prime}\right)
$$

which completes the proof of point (1).

The gauge symmetry is proven along the same line. 
We first consider Eq. (5.1). Since the free Schrödinger evolution fulfils gauge invariance, we have

$$
\int_{0}^{t} \mathrm{~d} s \frac{\left[U(s) \psi_{0}^{T}\right](0)}{\sqrt{t-s}}=\sqrt{\frac{T}{t+T}} \int_{0}^{t T /(t+T)} \mathrm{d} s\left[U(s) \psi_{0}\right](0)\left(\frac{T t}{T+t}-s\right)^{-1 / 2}
$$

and, from (5.1)

$$
\sqrt{\frac{t^{\prime}}{t}} q\left(t^{\prime}\right)+4 \sqrt{\frac{\pi \mathrm{i} t^{\prime}}{t}} \int_{0}^{t^{\prime}} \mathrm{d} s \frac{|q(s)|^{2} q(s)}{\sqrt{t^{\prime}-s}}=4 \sqrt{\pi \mathrm{i}} \int_{0}^{t} \mathrm{~d} s \frac{\left[U(s) \psi_{0}^{T}\right](0)}{\sqrt{t-s}},
$$

where we defined $t^{\prime} \equiv T t /(T+t)$.

Introducing the function $q^{T}$ defined as

$$
q^{T}(t) \equiv \sqrt{\frac{t^{\prime}}{t}} q\left(t^{\prime}\right)
$$

and using the change of variable $s^{\prime}=T s /(T-s)$ in the integral term in the 1.h.s. of Eq. (5.7), we show that $q^{T}$ solves Eq. (5.1) for the initial datum $\psi_{0}^{T}$.

Concerning the interaction term in formula (1.5) we notice that

$$
U\left(t-\frac{T s^{\prime}}{T-s^{\prime}} ; \mathbf{x}\right)=\left(\frac{T-s^{\prime}}{T+t}\right)^{3 / 2} \mathrm{e}^{\mathrm{i} \frac{|\mathbf{x}|^{2}}{4(T+t)}} U\left(\frac{T t}{T+t}-s^{\prime} ; \frac{T \mathbf{x}}{T+t}\right),
$$

and then

$$
\int_{0}^{t} \mathrm{~d} s U(t-s ; \mathbf{x}) q^{T}(s)=\left(\frac{T}{T+t}\right)^{3 / 2} \mathrm{e}^{\mathrm{i} \frac{|\mathbf{x}|^{2}}{4(T+t)}} \int_{0}^{T t /(T+t)} \mathrm{d} s^{\prime} U\left(\frac{T t}{T+t}-s^{\prime} ; \frac{T \mathbf{x}}{T+t}\right) q\left(s^{\prime}\right)
$$

which concludes the proof of Proposition 8.

Corollary 9 (Pseudo-conformal invariance). Under the same hypotheses of Proposition 8 the following symmetry law holds: if $\psi(t, \mathbf{x})$ is the solution assolciated to the initial datum $\psi_{0}(\mathbf{x})$, then

$$
\psi_{p c}^{T}(t, \mathbf{x})=\frac{\mathrm{e}^{-\mathrm{i} \frac{|\mathbf{x}|^{2}}{4(T-t)}}}{(T-t)^{3 / 2}} \psi\left(\frac{1}{T-t}, \frac{|\mathbf{x}|}{T-t}\right)
$$

is the solution associated to the initial datum

$$
\psi_{p c 0}^{T}(\mathbf{x})=\frac{\mathrm{e}^{-\mathrm{i} \frac{|\mathbf{x}|^{2}}{4 T}}}{T^{3 / 2}} \psi\left(\frac{1}{T}, \frac{|\mathbf{x}|}{T}\right) .
$$

Proof. The pseudo-conformal transformation can be seen as the composition of a dilation with a gauge transformation. Let us consider the initial datum $\psi_{1,0}(\mathbf{x})=\psi\left(T^{-1}, \mathbf{x}\right)$ and the corresponding solution $\psi_{1}(t, \mathbf{x})=$ $\psi\left(T^{-1}+t, \mathbf{x}\right)$. Applying a dilation to $\psi_{1,0}$ with parameter $T^{-1}$, we obtain $\psi_{2,0}(\mathbf{x})=T^{-3 / 2} \psi\left(T^{-1}, T^{-1} \mathbf{x}\right)$. The solution corresponding to the initial datum $\psi_{2,0}$ is then $\psi_{2}(t, \mathbf{x})=T^{-3 / 2} \psi\left(T^{-1}+T^{-2} t, T^{-1} \mathbf{x}\right)$. Now, it suffices to apply a gauge transformation with parameter $-T$ to $\psi_{2,0}$ to obtain $\psi_{p c 0}^{T}$, and to find that the corresponding solution is given by $\psi_{p c}^{T}$. 


\section{A class of explicit blow-up solutions}

Here we introduce a family of explicit blow-up solutions for Eqs. (1.5), (5.1). We follow the analogous contruction used for the standard NLSE (see, e.g., [6]), i.e., the explicit solutions are obtained starting from a family of stationary solutions and applying a pseudo-conformal transformation. In contrast with the standard case, for our system it is possible to write explicitely the entire family of stationary solutions.

Definition 10. A solution of the system (1.5), (5.1) of the form

$$
\psi_{s t}(t, \mathbf{x})=\mathrm{e}^{\mathrm{i} \omega t} \psi_{0, s t}(\mathbf{x}), \quad \omega \in \mathbb{R},
$$

is called a stationary solution.

The family of stationary solutions is characterized in the following proposition.

Proposition 11. The entire family of stationary solutions for the problem (1.5), (5.1) is given by

$$
\psi_{s t}^{\eta, \omega}=\mathrm{e}^{\mathrm{i} \eta} \mathrm{e}^{\mathrm{i} \omega t} \frac{\omega^{1 / 4}}{\sqrt{4 \pi|\gamma|}} \frac{\mathrm{e}^{-\sqrt{\omega}|\mathbf{x}|}}{4 \pi|\mathbf{x}|}
$$

with $\eta \in \mathbb{R}, \omega>0, \gamma<0$.

Proof. Since the evolution of the function $q$ is given by $q_{s t}(t)=\mathrm{e}^{\mathrm{i} \omega t} q_{0, s t}$, the problem for the stationary solutions reduces to

$$
\begin{aligned}
& \mathrm{e}^{\mathrm{i} \omega t} \psi_{0, s t}=U(t) \psi_{0, s t}+\mathrm{i} q_{0, s t} \int_{0}^{t} \mathrm{~d} s \mathrm{e}^{\mathrm{i} \omega s} U(t-s ; \cdot), \\
& \mathrm{e}^{\mathrm{i} \omega t} q_{0, s t}+4 \sqrt{\pi \mathrm{i}} \gamma\left|q_{0, s t}\right|^{2} q_{0, s t} \int_{0}^{t} \mathrm{~d} s \frac{\mathrm{e}^{\mathrm{i} \omega s}}{\sqrt{t-s}}=4 \sqrt{\pi \mathrm{i}} \int_{0}^{t} \mathrm{~d} s \frac{\left[U(s) \psi_{0, s t}\right](0)}{\sqrt{t-s}} .
\end{aligned}
$$

We notice that problem (6.3), (6.4) corresponds to the equations for the bound states for the linear problem, with a delta interaction whose strength equals $\alpha=\gamma\left|q_{0, s t}\right|^{2}$. For such a problem the family of bound states consists in the one dimensional linear space (see, e.g., [4])

$$
\psi_{s t}(t, \mathbf{x})=N \mathrm{e}^{\mathrm{i} \omega t} \frac{\mathrm{e}^{4 \pi \alpha|\mathbf{x}|}}{4 \pi|\mathbf{x}|}, \quad \alpha<0, \omega=16 \pi^{2} \alpha^{2}, N \in \mathbb{C} .
$$

In the nonlinear framework we have the additional condition $\alpha=\gamma\left|q_{0, s t}\right|^{2}$ which, put together with the relation $q_{0, s t}=N$, gives

$$
\psi_{s t}(t, \mathbf{x})=\mathrm{e}^{\mathrm{i} \omega t} \frac{\omega^{1 / 4}}{\sqrt{4 \pi|\gamma|}} \frac{\mathrm{e}^{-\sqrt{\omega}|\mathbf{x}|}}{4 \pi|\mathbf{x}|} .
$$

The uniqueness of the family of stationary solutions follows from the uniqueness of the family for the corresponding linear problem.

Remark 12. The same proof holds also for arbitrary $\sigma$. In that case, the entire family of stationary solution is given by

$$
\psi_{s t}(t, \mathbf{x})=\mathrm{e}^{\mathrm{i} \omega t}\left[\frac{\sqrt{\omega}}{4 \pi|\gamma|}\right]^{1 /(2 \sigma)} \frac{\mathrm{e}^{-\sqrt{\omega}|\mathbf{x}|}}{4 \pi|\mathbf{x}|}, \quad \omega>0 .
$$


Remark 13. The stationary solutions exhibited in Proposition 11 have the same $L^{2}$-norm and the same energy. In fact,

$$
\begin{aligned}
& \left\|\psi^{\eta, \omega}(t)\right\|_{L^{2}\left(\mathbb{R}^{3}\right)}=\left(32 \pi^{2}|\gamma|\right)^{-1}, \\
& E\left[\psi^{\eta, \omega}(t)\right]=0 .
\end{aligned}
$$

Applying the pseudo-conformal transformation to the family of stationary solutions exhibited in Proposition 11, we obtain the following result, whose proof is trivial.

Theorem 14. The following family of functions $\psi_{p c}^{\eta, \omega, T}$ consists of blow-up solutions for the problem (1.5), (5.1)

$$
\psi_{p c}^{\eta, \omega, T}(t, \mathbf{x})=\mathrm{e}^{\mathrm{i} \eta} \mathrm{e}^{\mathrm{i} \frac{\omega t}{T-t}} \frac{\omega^{1 / 4}}{\sqrt{4 \pi|\gamma|}} \frac{\mathrm{e}^{-\sqrt{\omega|\mathbf{x}| /(T-t)}}}{4 \pi \sqrt{T-t}|\mathbf{x}|} .
$$

Remark 15. The solutions given in Theorem 14 satisfy

$$
\begin{aligned}
& \left\|\psi_{p c}^{\eta, \omega, T}(t)\right\|_{L^{2}\left(\mathbb{R}^{3}\right)}=\left(32 \pi^{2}|\gamma|\right)^{-1}, \\
& E\left[\psi_{p c}^{\eta, \omega, T}(t)\right]=0 .
\end{aligned}
$$

Therefore these blow-up solutions do not fulfil the hypotheses of Theorem 7 . Indeed they only blow-up in one direction of time.

Notice that the quantity $\left(32 \pi^{2}|\gamma|\right)^{-1}$ is the minimal norm that a solution must possess in order to blow-up (see [1], Remark 4).

Remark 16. All the explicit blow-up solutions exhibited in Theorem 11 have the same blow-up rate. We observe that, in general, the characterization of the possible blow-up rates is an open problem.

Remark 17. It is also possible to prove the existence of solutions with $L^{2}$ norm larger than (6.11) which blow up only in one direction of time. Indeed, we start from a solution $\Xi(t)$ whose interval of existence is $\left(-T_{*}, T^{*}\right)$, with both $T_{*}$ and $T^{*}$ finite.

Now, we apply to the initial datum $\Xi_{0}$ a gauge transformation with parameter $T$, where $0<T<T^{*}$, obtaining the function $\Xi_{0}^{T}$. It is then immediately seen that the interval of existence for the solution $\Xi^{T}(t)$ associated to $\Xi_{0}^{T}$, is given by $\left(-T_{*} T /\left(T+T_{*}\right),+\infty\right)$.

\section{References}

[1] R. Adami, G. Dell'Antonio, R. Figari, A. Teta, The Cauchy problem for the Schrödinger equation in dimension three with a concentrated nonlinearity, Preprint, Département de mathématiques et applications, École normale supérieure, DMA-02-09, Ann. Inst. H. Poincaré Anal. Non Linéaire, in press.

[2] R. Adami, A. Teta, A simple model of concentrated nonlinearity, Operator Theory Adv. Appl. 108 (1999) 183-189.

[3] R. Adami, A. Teta, A class of nonlinear Schrödinger equation with concentrated nonlinearity, J. Funct. Anal. 180 (2001) 148-175.

[4] S. Albeverio, F. Gesztesy, R. Högh-Krohn, H. Holden, Solvable Models in Quantum Mechanics, Springer-Verlag, New York, 1988.

[5] T. Cazenave, An Introduction to Nonlinear Schrödinger Equation, second ed., in: Textos de Métodos Matematicos, vol. 26, IMUFRJ, Rio de Janeiro, 1993

[6] T. Cazenave, Blow-up and Scattering in the Nonlinear Schrödinger Equation, in: Textos de Métodos Matematicos, vol. 30, IMUFRJ, Rio de Janeiro, 1996

[7] J. Ginibre, G. Velo, On a class of nonlinear Schrödinger equations, I. The Cauchy problem, general case, J. Funct. Anal. 32 (1979) 1-32.

[8] T. Kato, On nonlinear Schrödinger equations, Ann. Inst. H. Poincaré Phys. Théor. 46 (1987) 113-129.

[9] F. Merle, Construction of solutions with exactly $k$ blow-up points for the Schrödinger equation with critical nonlinearity, Comm. Math. Phys. 129 (1990) 223-240.

[10] M.R. Sayapova, D.R. Yafaev, The evolution operator for time-dependent potentials of zero radius, Proc. Steklov Inst. Math. 2 (1984) 173-180.

[11] M.I. Weinstein, NLSE and sharp interpolation estimates, Comm. Math. Phys. 87 (1983) 567-576. 